\title{
An Example of Blowup for a Degenerate Parabolic Equation with a Nonlinear Boundary Condition
}

\author{
M. Chipot and J. Filo
}

\begin{abstract}
In this paper, a nonlinear parabolic equation of the form $u_{t}=\left(a\left(u_{x}\right)\right)_{x}$ for $x \in(0,1)$, $t>0, a\left(u_{x}\right)=\left|u_{x}\right|^{p-2} u_{x}$ if $u_{x} \geq \eta>0,1<p<2$, with nonlinear boundary condition $a\left(u_{x}(1, t)\right)=|u|^{p-2} u(1, t)$ is considered. It is proved that if $q p-3 p+2>0$, then the solutions blow up in finite timc. Morcover, estimates on the blowup profile (in $x$ ) and the blowup rate (in $t$ ) for $x=1$ are derived.
\end{abstract}

Keywords: Degencrate parabolic equalions, nonlinear boundary conditions, blowup

AMS subject classification: $35 \mathrm{~K} 65,35 \mathrm{~K} 55$

\section{Introduction}

In the recent years questions like blowup, global solvability and qualitative behaviour of solutions near blowup tine for semilinear parabolic problems have attracted considerable interest. Should we: restrict ourselves to problems with nonlinear boundary conditions, the heat equation has been discussed to a fair degree, however, nonlinear diffusion problems have not been studied in that extent (see [3] and references therein).

The purpose of this paper is to choose an interesting model problem to demonstrate how the diffusion coefficient, nonlinearly depending on the gradient of a solution, affects the value of criticial exponent for global solvability and to describe the behaviour of solutions that blow up in a finite time as detailed as possible. Thus, consider the problem

$$
\begin{aligned}
u_{t} & =\left(\left|u_{x}\right|^{p-2} u_{x}\right)_{x} \quad \text { in } \Omega_{T} \equiv(0,1) \times(0, T) \\
u_{s}(0, t) & =0 \\
\left|u_{x}(1, t)\right|^{p-2} u_{x_{x}}(1, t) & =|u(1, t)|^{q-2} u(1, t) \text { for } t \in(0, T) \\
u(\cdot, 0) & =\varphi \in W_{p}^{1}(0,1)
\end{aligned}
$$

M. Chipot: Inst. Angew. Mith., Univ. Zürich, Winterthurerstr. 190, CH -8057 Zürich, Switzerland

J. Filo: Inst. Appl. Mall.. ('omenius litiv., Mlynská dolina, 84215 Bratislava, Slovakia; the author has been partially supported by VEGA grant $1 / 4195 / 97$.

ISSN 0232-2064 / \$2.:0 (C) Helclermanu Verlag Berlin 
where $1<p<2$ and $q>1$ are given. The elementary inequality

$$
\begin{aligned}
\max _{0 \leq x \leq 1}|v(x)|^{(3 p-2) / p} \leq & \frac{3 p-2}{p}\left(\int_{0}^{1}\left|v_{x}(x)\right|^{p} d x\right)^{1 / p}\left(\int_{0}^{1}|v(x)|^{2} d x\right)^{(p-1) / p} \\
& +\int_{0}^{1}|v(x)|^{(3 p-2) / p} d x \quad \forall v \in W_{p}^{1}(0,1)
\end{aligned}
$$

with the help of Young's inequality easily yields

$$
|v(1)|^{(3 p-2) / p} \leq \varepsilon \int_{0}^{1}\left|v_{x}(x)\right|^{p} d x+C_{\varepsilon} \int_{0}^{1}|v(x)|^{2} d x+C
$$

for any $0<\varepsilon<\infty$. Using (1.2) it is not difficult to show (see Remark 1) that Problem (1.1) is globally solvable, i.e. it has a weak solution for arbitrarily large $T$ whenever

$$
q \leq \frac{3 p-2}{p}
$$

It is the aim of this note to show that the growth condition (1.3) for global solvability of problems like (1.1) is optimal.

To be more precise, let us take a function $a \in C^{4}(\mathbb{R})$,

$$
\begin{gathered}
a(\xi)=|\xi|^{p-2} \xi \quad \text { if }|\xi| \geq \eta>0 \\
a^{\prime}(\xi)>0 \text { and }|a(\xi)| \leq|\xi|^{p-1} \quad \text { for all } \xi \in \mathbb{R},
\end{gathered}
$$

$0<\eta \ll 1$ being arbitrary, but from now on fixed, and instead of (1.1) consider the problem

$$
\begin{aligned}
u_{t} & =\left(a\left(u_{x}\right)\right)_{x} & & \text { in } \Omega_{T} \\
u_{x}(0, t) & =0 & & \\
a\left(u_{x}(1, t)\right) & =|u(1, t)|^{q-2} u(1, t) & & \text { for } t \in[0, T) \\
u(x, 0) & =\varphi(x)>0 & & \text { for } x \in[0,1] .
\end{aligned}
$$

We would like to show that Problem (1.6) has for smooth $\varphi$ a classical solution which becomes unbounded in a finite time if

$$
q>\frac{3 p-2}{p}
$$

Moreover, we will derive estimates on the profile (in $x$ ) and the rate (in $t$ ) for $x=1$ of the solutions to problem (1.6) when they approach the blowup timc. The methods, that we apply, borrow matcrial from [3] and the references therein, nevertheless, their application to our problem seems to be not straightforward.

Before stating our main result, let us make precise some notion we use throughout the paper. By a classical solution or shortly by a solution of Problem (1.6) on $\Omega_{T}$ 
we mean a smooth function $u$, say $u \in C^{2,1}\left(\bar{\Omega}_{T}\right)$, such that all identities in (1.6) are satisfied. Put

$$
T_{\max } \equiv \sup \left\{T \mid \text { there exists a classical solution of Problem (1.6) on } \Omega_{T}\right\} .
$$

By a maximal solution of Problem (1.6) we shall understand a function $u: \bar{\Omega} \times$ $\left[0, T_{\max }\right) \longrightarrow \mathbb{R}$ such that $u$ is a solution of $(1.6)$ on $\Omega_{T}$ for any $T, T<T_{\max }$. If $T_{\max }=\infty$, we say that Problem (1.6) is globally solvable. On the other hand, if $T_{\max }<\infty$ and $\max _{x \in \bar{\Omega}}|u(x, t)| \longrightarrow \infty$ as $t \rightarrow T_{\max }$, we say that the solution $u$ blows up in a finite time.

\section{Main results}

The following theorem summarizes our knowledge of the behaviour of the solutions to Problem (1.6).

Theorem 1. Let $1<p<2, p q-3 p+2>0, \varphi^{\prime}(1)>\eta$ and assume that $u$ is a maximal positive solution of Problem (1.6) such that

$$
u_{t}, u_{x}, u_{x t} \text { are continuous and non-negative }
$$

on $[0,1] \times\left[0, T_{\max }\right)$. Then:

(i) The solution $u$ blows up in a finite time and the maximal time of existence, $T_{\max }$, can be estimated from above as follows:

$$
0<T_{\max } \leq \tau(\varphi(1))^{-(p q-3 p+2) /(p-1)}
$$

where $\tau \equiv p /(p q-3 p+2)$.

(ii) Moreover, if $q<2$, then there exist positive constants $m, C$ and for each $\varepsilon \in(0,1)$ a constant $c_{\varepsilon}$ such that

$$
\frac{c_{\varepsilon}}{\left(T_{\max }-t\right)^{(p-1) /(p q-3 p+2+c)}} \leq u(1, t) \leq \frac{C}{\left(T_{\max }-t\right)^{(p-1) /(p q-3 p+2)}}
$$

for all $t \in\left[0, T_{\max }\right)$, and

$$
\frac{m}{(1-x)^{(p-1) /(q-p)}} \leq u(x, t)
$$

for all $(x, t) \in[0,1] \times\left[0, T_{\max }\right)$ such that

$$
\zeta \leq x \leq 1-\Lambda^{\varepsilon}\left(T_{\max }-t\right)^{(q-p) /(p q-3 p+2+\varepsilon)},
$$

where $\zeta \equiv\left(1-(\sigma-1)^{-1}(\varphi(1))^{2-\sigma}\right)_{+}=\max \left\{0,1-(\sigma-1)^{-1}(\varphi(1))^{2-\sigma}\right\}, \Lambda^{\epsilon} \equiv(\sigma-$ $1)^{-1} c_{\varepsilon}^{2-\sigma}$ and

$$
\sigma-1=\frac{q-1}{p-1}
$$



that

(iii). On the other hand, if $q \geq 2$, then there exist positive constants $c, C, m, M$ such

$$
\frac{c}{\left(T_{\max }-t\right)^{(p-1) /(p q-3 p+2)}} \leq u(1, t) \leq \frac{C}{\left(T_{\max }-t\right)^{(p-1) /(p q-3 p+2)}}
$$

for all $t \in\left[0, T_{\max }\right)$ and

$$
\frac{m}{(1-x)^{(p-1) /(q-p)}} \leq u(x, t) \leq \frac{M}{(\min \{1-\rho, 1-x\})^{(p-1) /(q-p)}}
$$

for some $\rho, 0<\rho<1$, where the right-hand side inequality holds for all $(x, t) \in[0,1] \times$ $\left[0, T_{\max }\right)$ and the left-hand side inequality only for those $(x, t)$, that satisfy

$$
\zeta \leq x \leq 1-\Lambda\left(T_{\max }-t\right)^{(q-p) /(p q-3 p+2)}
$$

with $\Lambda \equiv(\sigma-1)^{-1} c^{2-\sigma}$ and $\zeta$ as above.

The constants $c_{\varepsilon}, c, C, m, M, \rho$ are specified in the proof below.

The next theorem says that it is possible to find initial functions such that (2.1) is satisfied for corresponding solutions of Problem (1.6).

Theorem 2. Assume that $\varphi$ is a positive smooth solution of the problem

$$
\left.\begin{array}{rl}
\left(a\left(v_{x}\right)\right)_{x} & =\lambda v^{(q-1) /(p-1)}(x) \quad(0<x<1) \\
v_{x}(0) & =0 \\
a\left(v_{x}(1)\right) & =v^{q-1}(1)
\end{array}\right\}
$$

for some $\lambda>0$ and such that $\varphi^{\prime}(1)>\eta$. Then there exists a unique maximal classical solution of Problem (1.6) such that $u_{t}, u_{x} \in C^{2,1}\left(\bar{\Omega}_{T}\right)$ for all $T \in\left[0, T_{\max }\right)$ and $u_{t}, u_{x}, u_{x t}$ are positive on $(0,1] \times\left[0, T_{\max }\right)$.

Finally, the following theorem guarantees the classical solvability of Problem (2.10).

Theorem 3. Let $\mathcal{L}^{q-1}>\eta^{p-1}$ be given. Then there exists a positive function $v \in C^{\infty}([0,1]), v(1)=\mathcal{L}$ such that $v$ is a solution of Problem (2.10) with

$$
\lambda=\frac{\sigma A\left(\mathcal{L}^{\sigma-1}\right)}{\mathcal{L}^{\sigma}-\mu^{\sigma}}
$$

where $A(\xi)=\int_{0}^{\xi} a^{\prime}(\tau) \tau d \tau$ and $\mu=v(0)$. 


\section{Proof of Theorem 1}

1. First of all note that our assumption $\varphi^{\prime}(1)>\eta$ yields $a\left(u_{x}(1, t)\right)=u_{x}^{p-1}(1, t)$ for all $t \in\left[0, T_{\max }\right)$ and one can compute

$$
u^{p(\sigma-1)}(1, t)=u_{x}^{p}(1, t)=a\left(u_{x}(1, t)\right) u_{x}(1, t)=\int_{0}^{1}\left(\left(a\left(u_{x}\right)\right)_{x} u_{x}+a\left(u_{x}\right) u_{x x}\right) d x
$$

As $\left(a\left(u_{x}\right)\right)_{x}=u_{t}$, integrating the first term on the right-hand side of the above identity by parts we arrive at

$$
u^{p(o-1)}(1, t)=u_{t}(1, t) u(1, t)-u_{t}(0, t) u(0, t)-\int_{0}^{1} u_{x t} u d x+\int_{0}^{1} a\left(u_{x}\right) u_{x x} d x .
$$

In view of (1.5) and (2.1) we have

$$
\int_{0}^{1} a\left(u_{x}\right) u_{x x} d x \leq \int_{0}^{1} u_{x}^{p-1} u_{x x} d x
$$

that with the previous inequality yield

$$
u^{p(o-1)}(1, t) \leq u_{t}(1, t) u(1, t)+p^{-1} \int_{0}^{1}\left(u_{x}^{p}\right)_{x} d x
$$

i.e.

$$
u_{t}(1, t) \geq \alpha u^{1+\theta}(1, t),
$$

where $\alpha \equiv \frac{p-1}{p}$ and $\theta \equiv \frac{p q-3 p+2}{p-1}$. Solving this differential inequality one can see that

$$
T_{\max } \leq T_{*} \equiv \frac{1}{\alpha \theta(\varphi(1))^{\theta}} \quad \text { and } \quad \frac{\varphi(1)}{\left(1-T_{*}^{-1} t\right)^{1 / \theta}} \leq u(1, t)
$$

for all $t \in\left[0, T_{\max }\right.$ ). Now it is not difficult to show that $u(1, t) \rightarrow \infty$ if $t \rightarrow T_{\max }$ (see the proof of Theorem 2 below). Next, integrate (3.1) over $(s, t), s<t$ :

$$
-(u(1, s))^{-\theta} \leq(u(1, t))^{-\theta}-(u(1, s))^{-\theta} \leq-\alpha \theta(t-s) .
$$

For $t$ going to $T_{\max }(3.2)$ easily yields $(2.3)_{2}$ and $(2.7)_{2}$ with $C \equiv(\alpha \theta)^{-1 / \theta}$. Note that by the notation $(2.3)_{2}$ we understand the second inequality in (2.3), i.e.

$$
u(1, t) \leq \frac{C}{\left(T_{\max }-t\right)^{(p-1) /(p q-3 p+2)}} .
$$

2. Now choose $0<\rho<1, n \geq 2$, define $\chi(x) \equiv\left(\frac{(x-\rho)_{t}}{1-\rho}\right)^{n}$ and write

$$
J(x, t) \equiv u_{x}(x, t)-\chi(x) u^{\sigma-1}(x, t) .
$$


We now claim that if $\rho$ is close enough to 1 and $n$ is sufficiently large, then

$$
J(x, t) \geq 0 \quad \text { on }[0,1] \times\left[0, T_{\max }\right)
$$

if $q \geq 2$. In fact, observe first that due to our assumptions $u_{x}(1,0)=\varphi^{\prime}(1)>\eta, u_{x t} \geq 0$, we have $u_{x}(x, t) \geq \eta$ on $[\mu, 1] \times\left[0, T_{\max }\right)$ for some $0<\mu<1$. Taking $\rho \geq \mu$ it is not difficult to verify that $J$ satisfies the following set of equations:

$$
\begin{aligned}
\mathcal{P} J \equiv & J_{t}-a^{\prime}\left(u_{x}\right) J_{x x}+A(x, t) J_{x}+B(x, t) J \\
= & (p-1) u_{x}^{p-3} u^{2(\sigma-1)}\left(\chi \chi^{\prime \prime}-(2-p)\left(\chi^{\prime}\right)^{2}\right. \\
& \left.+2(q-1) u^{\sigma-2} \chi^{\prime} \chi^{2}+(\sigma-1)(q-2) u^{2 \sigma-4} \chi^{4}\right) \text { on } \Omega_{T} \\
J(0, t)= & J(1, t)=0 \text { for } t \in[0, T] \\
J(x, 0)= & \varphi^{\prime}(x)-\chi(x) \varphi^{\sigma-1}(x) \quad \text { for } x \in[0,1]
\end{aligned}
$$

for some bounded and continuous functions $A$ and $B$, and for any $T<T_{\max }$. Now, if

$$
q \geq 2 \quad \text { and } \quad n \geq \max \left\{2,(p-1)^{-1}\right\}
$$

then it is easy to verify that $\mathcal{P} J \geq 0$ on $\bar{\Omega}_{T}$. We next claim if $\rho$ is close enough to 1 ,

$$
J(x, 0) \geq 0 \quad \text { for } \quad x \in[0,1] .
$$

To see this, we note that due to $(2.1), \varphi^{\prime}, \varphi^{\prime \prime}, \varphi^{\prime \prime \prime} \geq 0$ on $[0,1]$. Then

$$
\varphi^{\prime}(x) \geq \ell(x) \equiv\left(\varphi^{\prime \prime}(1)(x-1)+(\varphi(1))^{\sigma-1}\right)_{+} .
$$

This for $x=0$ yields $\varphi^{\prime \prime}(1) \geq(\varphi(1))^{\sigma-1}$ and one can conclude that $\ell(x) \equiv 0$ for all $x \in\left[0, x_{0}\right], x_{0}=1-(\varphi(1))^{\sigma-1} / \varphi^{\prime \prime}(1)$. Taking $\rho \geq x_{0}$ and making use the convexity of the function $\chi(x) \varphi^{\sigma-1}(x)$ together with the fact that $\varphi^{\sigma-1}(1)=\ell(1)$ and $\chi\left(x_{0}\right) \varphi^{\sigma-1}\left(x_{0}\right)=$ $\ell\left(x_{0}\right)=0$ we arrive at

$$
\chi(x) \varphi^{\sigma-1}(x) \leq \ell(x) \quad \text { for all } x \in[0,1]
$$

and (3.7) follows. Thus, the maximum principle yields (3.4), and so the estimate $(2.8)_{2}$ follows by integration. Indeed, integrating $(3.4)$ over $(x, 1)$ we find

$$
u^{\sigma-2}(x, t) \leq 1 /(\sigma-2) g(x), \quad g(x) \equiv \int_{x}^{1} \chi(\tau) d \tau
$$

Note $g \in C^{3}, g(x)=\int_{\rho}^{1} \chi(\tau) d \tau$ for all $x \in[0, \rho], g(1)=0$ and $g^{\prime \prime}(x) \leq 0$. Thus

$$
g(x) \geq \int_{\rho}^{1} \chi(\tau) d \tau \min \left\{1, \frac{x-1}{\rho-1}\right\}
$$


and

$$
u(x, t) \leq\left[\frac{\sigma-2}{1-\rho} \int_{\rho}^{1} \chi(\tau) d \tau\right]^{1 /(2-\sigma)} \frac{1}{(\min \{1-\rho, 1-x\})^{(p-1) /(q-p)}}
$$

for all $x \in[0,1]$, i.e. $(2.8)_{2}$.

3. In view of (3.4), i.e. $J(x, t) \geq 0$ and due to the fact that $J(1, t)=0$ we find $J_{z}(1, t) \leq 0$, i.e.

$$
u_{t}(1, t) \leq(p-1) \chi^{\prime}(1) u^{(p-1)(\sigma-1)}(1, t)+(q-1) u^{p(\sigma-1)-1}(1, t)
$$

where $\chi^{\prime}(1)=n /(1-\rho)$. Note that $p(\sigma-1)-1-(p-1)(\sigma-1)=\sigma-2>0$. Therefore

$$
u_{t}(1, t) \leq\left((p-1) \chi^{\prime}(1)+q-1\right) u^{p(\sigma-1)-1}(1, t) \quad \forall t \in\left[\iota, T_{\max }\right),
$$

where $\iota \equiv \min \left\{s \mid s \in\left[0, T_{\max }\right), u(1, s) \geq 1\right\}$. Consequently,

$$
(u(1, t))^{-\theta}-(u(1, s))^{-\theta} \geq-\left(q-1+(p-1) \chi^{\prime}(1)\right) \theta(t-s) \quad \forall t, s \in\left[\iota, T_{\max }\right) .
$$

Thus, after $t \rightarrow T_{\max }\left(\lim _{t \rightarrow T_{\max }} u(1, t)=\infty\right)$, we arrive at

$$
u(1, t) \geq\left(\frac{1}{\left(q-1+(p-1) \chi^{\prime}(1)\right) \theta\left(T_{\max }-t\right)}\right)^{1 / \theta}
$$

for all $t \in\left[i, T_{\max }\right)$, and $(2.7)_{1}$ follows.

4. To obtain the estimate for $q<2$ we have to proceed differently. Put

$$
y(t)=u^{\nu}(1, t) \int_{1-\xi(t)}^{1} u(x, t) d x, \xi(t)=u^{-\omega}(1, t), \omega=\frac{q-p}{p-1}+\varepsilon, \nu=\omega-1+\varepsilon .
$$

Then

$$
y^{\prime}(t)=-u^{\nu-1}(1, t) u_{t}(1, t) I(t)+u^{\nu}(1, t) \int_{1-\xi(t)}^{1}\left(a\left(u_{x}\right)\right)_{x} d x
$$

where

$$
I(t)=\omega u(1-\xi(t), t) \xi(t)-\nu \int_{1-\xi(t)}^{1} u(x, t) d x .
$$

Let us first consider the more difficult case $\nu>0$. Below we shall use the following estimate, which follows from the non-negativeness of $u_{x}, u_{x x}$ and from the form of our boundary condition at $x=1$ :

$$
u(1, t)\left(1-u^{-\varepsilon}(1, t)\right) \leq u(1-\xi(t), t) \leq u(x, t) \leq u(1, t)
$$

for all $x \in[1-\xi(t), 1], t \in\left[0, T_{\max }\right)$, that by integration yields

$$
u^{\varepsilon}(1, t)-1 \leq y(t) \leq u^{\varepsilon}(1, t)
$$


Now we show that there exists $\tau \geq 0$ such that $I(t) \geq 0$ for $t \geq \tau$. As

$$
\begin{aligned}
I(t) & =(1-\varepsilon) \int_{1-\xi(t)}^{1} u(x, t) d x-\omega \int_{1-\xi(t)}^{1}(u(x, t)-u(1-\xi(t), t)) d x \\
& \geq(1-\varepsilon) \int_{1-\xi(t)}^{1} u(x, t) d x-\omega(u(1, t)-u(1-\xi(t), t)) \xi(t)
\end{aligned}
$$

applying (3.11) we arrive at

$$
I(t) \geq u^{1-\varepsilon}(1, t) \xi(t)(1-\varepsilon)\left[u^{\varepsilon}(1, t)-\frac{\sigma-1}{1-\varepsilon}\right]
$$

which is positive if $t$ is sufficiently large. Note that $\sigma-1>1-\varepsilon$. Thus, (3.10) yields

$$
y^{\prime}(t) \leq u^{\theta+2 \varepsilon}(1, t), \quad \theta=(q p-3 p+2) /(p-1)
$$

which with the assistance of (3.12) gives

$$
z^{\prime}(t) \leq z^{1+(\theta+\varepsilon) / \varepsilon}(t), \quad z(t)=y(t)+1
$$

Now, if we take $t, s \in[\tau, T)$ such that $s<t$, by integrating of (3.13) we get

$$
z^{-\delta}(s)-z^{-\delta}(t) \leq \delta(t-s), \quad \delta=\frac{\theta+\varepsilon}{\varepsilon}=\frac{p q-3 p+2+\varepsilon(p-1)}{\varepsilon(p-1)}
$$

Next, (3.13) and (3.12) yield

$$
\frac{1}{2^{\delta} u^{\varepsilon \delta}(1, s)} \leq \frac{1}{u^{\varepsilon \delta}(1, t)}+\delta(t-s)
$$

that by letting $t \rightarrow T_{\max }$ gives

$$
\frac{1}{\left(2^{\delta} \delta\left(T_{\max }-s\right)\right)^{(p-1) /(p q-3 p+e(p-1))}} \leq u(1, s) .
$$

Hence, $(2.3)_{1}$ follows. In the case of $\nu \leq 0, I(t) \geq 0$ and the rest of the proof is the same as above.

5. Now, at last, we show the validity of (2.4) and (2.8) . We know from (2.1) that

$$
u(x, t) \geq u(x, s) \geq u_{x}(1, s)(x-1)+u(1, s)=u^{\sigma-1}(1, s)(x-1)+u(1, s)
$$

for any $s \in[0, t]$. Consequently,

$$
u(x, t) \geq \max _{\varphi(1) \leq \alpha \leq u(1, t)}\left(\alpha^{\sigma-1}(x-1)+\alpha\right) \geq \max _{\varphi(1) \leq \alpha \leq \vartheta(t)}\left(\alpha^{\sigma-1}(x-1)+\alpha\right),
$$


where $\vartheta(t) \equiv d\left(T_{\max }-t\right)^{-\beta}$ and

$$
d \equiv\left\{\begin{array} { l l } 
{ c _ { \varepsilon } } & { \text { if } q < 2 } \\
{ c } & { \text { if } q \geq 2 }
\end{array} \quad \text { and } \quad \beta \equiv \left\{\begin{array}{ll}
\frac{p-1}{p q-3 p+2+\varepsilon} & \text { if } q<2 \\
\frac{p-1}{p q-3 p+2} & \text { if } q \geq 2 .
\end{array}\right.\right.
$$

Note that for fixed $0<x<1$

$$
\max _{0 \leq \alpha<\infty}\left(\alpha^{\sigma-1}(x-1)+\alpha\right)=\left.\left(\alpha^{\sigma-1}(x-1)+\alpha\right)\right|_{\alpha=\alpha_{0}}=\frac{m}{(1-x)^{(p-1) /(q-p)}}
$$

where

$$
\alpha_{0}=[(\sigma-1)(1-x)]^{1 /(2-\sigma)} \quad \text { and } \quad m=(\sigma-2)(\sigma-1)^{(1-\sigma) /(\sigma-2)} \text {. }
$$

Observe next that $\varphi(1) \leq \alpha_{0} \leq \vartheta(t)$ whenever

$$
1-\frac{p-1}{q-1}(\varphi(1))^{-(q-p) /(p-1)} \leq x \leq 1-\frac{p-1}{q-1}(\vartheta(t))^{-(q-p) /(p-1)} .
$$

Hence, $u(x, t) \geq m(1-x)^{(1-p) /(q-p)}$ if $(3.16)$ holds.

\section{Proof of Theorem 2}

Let $u_{0}$ be a positive smooth solution of Problem (2.10) and denote $\mu=u_{0}(0), \mathcal{L}=u_{0}(1)$. Let $\phi \in C^{3}(\mathbb{R}), \phi(0)=0, \phi_{u} \leq 0,\left|\phi, \phi_{u}, \phi_{u}\right| \leq C$ on $\mathbb{R}$ for some positive constant $C$ and

$$
\phi(u)=-|u|^{q-2} u \quad \text { if } 0<\mu \leq u \leq M(=2 \mathcal{L}) .
$$

Morcover, let $a_{1} \in C^{4}(\mathbb{R}), 0<\varrho \leq a_{1}^{\prime}(\xi) \leq \varrho^{-1}$ on $\mathbb{R}$ for some $0<\varrho<1$ and

$$
a_{1}(\xi)=a(\xi) \quad \text { if }|\xi| \leq K\left(=M^{\sigma-1}\right),
$$

and consider the following problem:

$$
\begin{aligned}
u_{t}-\left(a_{1}\left(u_{x}\right)\right)_{x} & =0 & & \text { in } \Omega_{T} \\
a_{1}\left(u_{x}(1, t)\right)+\phi(u(1, t))=0, u_{x}(0, t) & =0 & & \text { on }[0, T] \\
u(x, 0) & =u_{0}(x) & & \text { on }[0,1] .
\end{aligned}
$$

Now, the results of [10: Chapter V/p. 429] guarantees the existence of a unique solution to Problem (4.1) in the class $H^{2+\beta, 1+\beta / 2}\left(\bar{\Omega}_{T}\right)$ for any $0<\beta<1$. However, to show that $u_{t}, u_{x}, u_{x x}, u_{x t}$ are non-negative we first show that $u$ is indeed more regular. To do this we rewrite Problem (4.1) into the following form:

$$
\begin{aligned}
u_{1}-\alpha(x, t) u_{x x} & =0 & & \text { in } \Omega_{T} \\
u_{x}(1, t)+b(t) u(1, t)=0, u_{x}(0, t) & =0 & & \text { on }[0, T] \\
u(x, 0) & =u_{0}(x) & & \text { on }[0,1] .
\end{aligned}
$$


where

$$
\alpha(x, t)=a_{1}^{\prime}\left(u_{x}(x, t)\right) \quad \text { and } \quad b(t)=-\frac{a_{1}^{-1}(-\phi(u(1, t)))}{u(1, t)} .
$$

As $D_{x}^{m} \alpha(m=0,1)$ are Hölder continuous in $\bar{\Omega}_{T}$, by [7: Chapter 3, Section $5 /$ Theorem 10] we obtain that $D_{x}^{m} u, D_{t} D_{x}^{k} u \quad(0 \leq m \leq 3,0 \leq k \leq 1)$ exist and are Hölder continuous in $\Omega_{T}$. Hence, the equation in (4.1) can be differentiated with respect to $x$ and for $w=u_{x}$ we obtain

$$
\begin{aligned}
w_{t}-a_{1}^{\prime}\left(u_{x}\right) w_{x x}-a_{1}^{\prime \prime}\left(u_{x}\right) u_{x x} w_{x} & =0 & & \text { in } \Omega_{T} \\
w(1, t)=a_{1}^{-1}(-\phi(u(1, t)), w(0, t) & =0 & & \text { on }[0, T] \\
w(x, 0) & =u_{0}^{\prime}(x) & & \text { on }[0,1] .
\end{aligned}
$$

By [10: Chapter IV/Theorem 5.2] it has a unique solution $w \in H^{2+\beta, 1+\beta / 2}\left(\bar{\Omega}_{T}\right)$. Thus, $D_{x}^{m} D_{t}^{k} \alpha \quad(0 \leq m+2 k \leq 2, k \leq 1)$ exist and are Hölder continuous in $\bar{\Omega}_{T}$ and by [7: Chapter 3, Section 5/Theorem 11] for (4.2) we arrive at $D_{x}^{m} D_{t}^{k} u(0 \leq m+2 k \leq 4, k \leq 2)$ to be Hölder continuous in $\Omega_{T}$. As, moreover, $u_{x t} \in C\left(\bar{\Omega}_{T}\right)$, we can differentiate the equation in (4.1) with respect to $t$, and the boundary condition too. Putting $U=u_{t}$ one can check that

$$
\begin{aligned}
U_{t}-a_{1}^{\prime}\left(u_{x}\right) U_{x x}-a_{1}^{\prime \prime}\left(u_{x}\right) u_{x x} U_{x} & =0 & & \text { in } \Omega_{T} \\
a_{1}^{\prime}\left(u_{x}\right) U_{x}(1, t)+\phi^{\prime}(u) U(1, t)=0, a_{1}^{\prime}\left(u_{x}\right) U_{x}(0, t) & =0 & & \text { on }[0, T] \\
U(x, 0) & =\left(a_{1}\left(u_{0}^{\prime}\right)\right)^{\prime}(x) & & \text { on }[0,1] .
\end{aligned}
$$

Here, the hypotheses of [10: Chapter IV/Theorem 5.2] are fulfilled and we obtain $U \in$ $H^{2+\beta, 1+\beta / 2}\left(\bar{\Omega}_{T}\right)$. Thus, similarly as above, one can conclude that $D_{x}^{m} D_{t}^{k} u$ exist and are Hölder continuous in $\Omega_{T}$ for $0 \leq m+2 k \leq 6, k \leq 3$. Hence, $W=u_{x t}$ satisfies

$$
\begin{aligned}
& W_{t}=a_{1}^{\prime}\left(u_{x}\right) W_{x x}+\left(a_{1}^{\prime \prime}\left(u_{x}\right) u_{x x x}+a_{1}^{\prime \prime \prime}\left(u_{x}\right) u_{x x}^{2}\right) W+2 a^{\prime \prime}\left(u_{x}\right) u_{x x} W_{x} \text { in } \Omega_{T} \\
& a_{1}^{\prime}\left(u_{x}\right) W(1, t)+\phi^{\prime}(u) U(1, t)=0, a_{1}^{\prime}\left(u_{x}\right) W(0, t)=0 \quad \text { on }[0, T] \\
& W(x, 0)=\left(a_{1}\left(u_{0}^{\prime}\right)\right)^{\prime \prime}(x) \quad \text { on }[0,1] .
\end{aligned}
$$

After these preliminaries it is not difficult to prove Theorem 2. Indeed, as $u_{t}(x, 0)=$ $\lambda u_{0}^{\sigma-1}(x) \geq \lambda \mu^{\sigma-1}>0$, the maximum principle yields that $u_{t}(x, t)>0$ on $\bar{\Omega}_{T}$. Thus, $u(x, t) \geq \mu$ on $\bar{\Omega}_{T}$ and the equation in (4.2) gives $u_{x x}>0$ on $\bar{\Omega}_{T}$. Now, as $u_{x t}(x, 0)=$ $\lambda(\sigma-1) u_{0}^{\sigma-2}(x) u_{0}^{\prime}(x)>0$ on $(0,1]$, taking into account (4.5) one can conclude that $u_{x t}(x, t)>0$ on $(0,1] \times[0, T]$.

Next, because of the smoothness of $u$ and due to the fact that $u_{0}(x) \leq \mathcal{L}=M / 2$ there exists a time $s>0$ such that $u(x, t) \leq M$ on $\bar{\Omega}_{s}$, i.e. $u$ is also the unique solution of our original problem (1.6) such that

$$
u, u_{t}, u_{x x}>0 \text { on } \bar{\Omega}_{s} \quad \text { and } \quad u_{x}, u_{x t}>0 \text { on }(0,1] \times[0, s] .
$$

Put

$$
T_{\text {max }}=\sup \left\{s \mid \text { there is a solution of Problem (1.6) on } \bar{\Omega}_{s}\right\} .
$$


If $T_{\max }<\infty$, it is not difficult to show that $u(1, t) \rightarrow \infty$ if $t \rightarrow T_{\max }$. Indeed, let us suppose that $u(1, t) \leq$ const for all $t \in\left[0, T_{\max }\right)$. Then from (4.1), (4.3) and (4.4) one can get

$$
u_{x}(x, t)+u_{t}(x, t)+u_{x x}(x, t) \leq C<\infty
$$

for all $(x, t) \in[0,1] \times\left[0, T_{\max }\right)$. Note that each term on the left-hand side of $(4.6)$ is non-negative. Applying now [10: Chapter IV/Theorem 5.3] for Problem (4.2) and then [10: Chapter IV/Theorem 5.2] for (4.3) we obtain the uniform bound of $u, u_{x}$ in the norm of $H^{2+\beta, 1+\beta / 2}\left(\bar{\Omega}_{T}\right)$ with respect to $T, T<T_{\max }$. Denote

$$
v(x) \equiv \lim _{t \rightarrow T_{\max }} u(x, t) \text {. }
$$

Then $v \in H^{2+\nu}([0,1]) \quad(0<\nu<1), v_{x}(0)=0$ and $a\left(v_{x}(1)\right)=v^{q-1}(1)$, i.e. the solution $u$ can be extended beyond $T_{\max }$, which is a contradiction.

\section{Proof of Theorem 3}

We shall first deal with the problem

$$
\left.\begin{array}{rl}
\left(a_{2}\left(v_{x}\right)\right)_{x} & =\lambda v^{\sigma-1} \quad(0<x<1) \\
v_{x}(0) & =0 \\
a_{2}\left(v_{x}(1)\right) & =v^{q-1}(1) .
\end{array}\right\}
$$

for some $\lambda>0$, where $a_{2} \in C^{3}(\mathbb{R}), 0<\kappa \leq a_{2}^{\prime}(\xi) \leq \kappa^{-1} \quad(\xi \in \mathbb{R})$ for some $0<\kappa<1$ and $a_{2}(\xi)=a(\xi)$ if $|\xi| \leq 2 \mathcal{L}^{\sigma-1}$. Put $\mathcal{A}(\xi)=\int_{0}^{\xi} a_{2}^{\prime}(\tau) \tau d \tau$ and denote by $\mathcal{A}^{-1}$ its inverse (on $[0, \infty)$ ). Then we have $\kappa \xi^{2} \leq 2 \mathcal{A}(\xi) \leq \kappa^{-1} \xi^{2}$ and

$$
'(2 \kappa)^{1 / 2} \sqrt{\xi} \leq \mathcal{A}^{-1}(\xi) \leq\left(\frac{2}{\kappa}\right)^{1 / 2} \sqrt{\xi}
$$

for any $\xi \in \mathbb{R}^{+}$. If $v$ is a positive classical solution of Problem (5.1), then $v_{x}>0$ on $(0,1]$ and multiplying the equation in $(5.1)$ by $v_{x}$ we obtain easily

$$
v_{x}(x)=\mathcal{A}^{-1}\left(\frac{\lambda}{\sigma}\left(v^{\sigma}(x)-\mu^{\sigma}\right)\right)
$$

where $\mu=v(0)$. Denoting $\ell=v(1), \hat{\ell}=\ell^{\sigma-1}$ and assuming that $\hat{\ell} \geq \eta,(5.3)$ for $x=1$ gives

$$
\lambda=\frac{\sigma \mathcal{A}(\hat{\ell})}{\ell^{\sigma}-\mu^{\sigma}} .
$$

After integration of (5.3) we arrive at

$$
\int_{\mu}^{v(x)} \frac{d z}{\mathcal{A}^{-1}\left(\frac{\lambda}{\sigma}\left(z^{\sigma}-\mu^{\sigma}\right)\right)}=x \quad \forall x \in[0,1] .
$$

Putting

$$
\mathcal{F}(\ell, \mu)=\int_{\mu}^{\ell} \frac{d z}{\mathcal{A}^{-1}\left(\frac{\lambda}{\sigma}\left(z^{\sigma}-\mu^{\sigma}\right)\right)}
$$

(5.5) for $x=1$ especially yields

$$
\mathcal{F}(\ell, \mu)=1 \text {. }
$$

Now we are ready to formulate the following two statements. 
Proposition 1. Assume that $\hat{\ell} \geq \eta$, let constants $0<\mu<\ell$ be related by equation (5.6) and a function $v$ be given implicitly by (5.5). Then $v \in C^{\infty}([0,1])$ and $v$ is a positive solution of Problem (5.1) with $v(0)=\mu, v(1)=\ell$ and $\lambda$ being given by (5.4).

Proposition 2. Let $\hat{\ell} \geq \eta$ be given. Then there exist a positive $\lambda$ and $\mu \in(0, \ell)$ such that (5.6) is satisfied.

Proof of Proposition 1. Let $0<\mu<\ell$ be given such that (5.6) is satisfied with $\lambda$ being given by (5.4). Put

$$
h(y)=\int_{\mu}^{y} \frac{d z}{\mathcal{A}^{-1}\left(\mathcal{A}(\hat{\ell})\left(\frac{z^{\sigma}-\mu^{\sigma}}{\ell^{\sigma}-\mu^{\sigma}}\right)\right)} \quad \text { for } y \in(\mu, \infty) .
$$

With the assistance of (5.2) one can now obtain for the function $h$ the estimate

$$
\left(\frac{\kappa}{2}\right)^{1 / 2} F(y) \leq h(y) \leq(2 \kappa)^{-1 / 2} F(y)
$$

where

$$
F(y)=\frac{\mu}{\sqrt{\mathcal{A}(\hat{\ell})}} f\left(\frac{y}{\mu}\right) \sqrt{\left(\frac{\ell}{\mu}\right)^{\sigma}-1}
$$

and

$$
f(x)=\int_{1}^{x} \frac{d z}{\sqrt{z^{\sigma}-1}} \quad \text { for } x \in(1, \infty)
$$

The above integral converges since $\sqrt{z^{\sigma}-1} \sim \sqrt{\sigma(z-1)}$ near $z=1$, hence, $h$ is well defined on $[\mu, \infty), h \in C([\mu, \infty)) \cap C^{\infty}(\mu, \infty), h(\mu)=0$ and $h(\ell)=1$. As $h^{\prime}(y)>0$ on $(\mu, \infty)$, for each $x \in[0,1]$ there exists a unique $y \in[\mu, \ell]$ which we shall denote $y=v(x)$ such that $v=h^{-1} \in C([0,1])$. As $v_{x}(x)=1 / h^{\prime}(v(x))$, i.e. (5.3), it is not difficult to see that $v$ satisfies (5.1) with $\lambda$ given by (5.4). Thus $a_{1}^{\prime}\left(v_{x}\right) v_{x x}=\lambda v^{\sigma-1}(x)$ and the smoothness of $v$ follows.

Proof of Proposition 2. Assume that $\ell$, satisfying $\hat{\ell} \geq \eta$, is given and put $z=\ell / \mu$. As we consider $0<\mu<\ell, z \in(1, \infty)$, and take

$$
W(z)=\frac{\ell z^{\sigma / 2-1}}{\sqrt{\mathcal{A}(\hat{\ell})}} f(z) \sqrt{1-\frac{1}{z^{\sigma}}}
$$

where $f$ is defined by (5.8). With the assistance of (5.7) one can see that

$$
\left(\frac{\kappa}{2}\right)^{1 / 2} W(z) \leq \mathcal{F}(\ell, \ell / z) \leq(2 \kappa)^{-1 / 2} W(z) \quad \text { for } z \in(1, \infty) \text {. }
$$

We know already that $W(z) \rightarrow 0$ as $z \rightarrow 1$ and it is enough to show that $W(z) \rightarrow \infty$ as $z \rightarrow \infty$, because then (5.10) yields the existence of at least one $z_{0} \in(1, \infty)$ such that $\mathcal{F}\left(\ell, \ell / z_{0}\right)=1$, and the assertion of Proposition 2 would follow. The fact that $W(z) \rightarrow \infty$ as $z \rightarrow \infty$ follows from (5.9) taking into account the fact that $\sigma>2$.

Proof of Theorem 3. Put $\ell=\mathcal{L}$. As $v_{x x}>0$ on $[0,1]$ it follows that $0 \leq v_{x}(x) \leq$ $\mathcal{L}^{\sigma-1}$ for all $x \in[0,1]$. Since $a_{2}(\xi)=a(\xi)$ for $|\xi| \leq 2 \mathcal{L}^{\sigma-1}$ (see (5.1) above), the proof of Theorem 3 is completed. 


\section{Concluding remarks}

Let us finish the paper with some rcmarks concerning Problem (1.6) for those values of the parameters $p$ and $q$ that we do not concern here.

Remark 1. Let $q$ satisfy (1.3), i.e. the inequality

$$
|v(1)|^{q} \leq \varepsilon \int_{0}^{1}\left|v_{x}(x)\right|^{p} d x+C_{\varepsilon} \int_{0}^{1}|v(x)|^{2} d x+C
$$

holds for any $v \in W_{p}^{1}(0,1)$. Multiplying the equation in (1.6) by $u$ and taking into account $(6.1)$ and the fact that $c|\xi|^{p}-C \leq a(\xi) \xi \leq|\xi|^{p} \quad(\xi \in \mathbb{R})$ for some positive constants $c$ and $C$, one can easily arrive at .

$$
\max _{0 \leq t \leq T} \int_{0}^{1}|u(x, t)|^{2} d x \leq\left(\int_{0}^{1}\left|u_{0}(x)\right|^{2} d x+L\right) e^{K t}-L
$$

where $K, L>0$ do not depend on $u$. Now, from the energy inequality

$$
\int_{0}^{t} \int_{0}^{1} u_{t}^{2}(x, t) d x d t+J(u(t)) \leq J\left(u_{0}\right), \quad J(v)=\int_{0}^{1} \int_{0}^{v_{x}} a(\tau) d \tau d x-q^{-1}|v(1)|^{q}
$$

we obtain

$$
\int_{0}^{T} \int_{0}^{1} u_{t}^{2}(x, t) d x d t+\max _{0 \leq t \leq T}\|u(t)\|_{w_{p}^{1}(0,1)} \leq \text { const }
$$

which does not depend on $u$. Therefore, one can conclude that $u$ exists globally.

Remark 2. If $p \geq 2$ and $q \leq 2$, it is shown in [6] by the same way as outlined in Remark 1 that all solutions of Problems (1.1) and (1.6) exist globally. In the case $q>2$ all non-decreasing (in $x$ ) positive solutions of Problem (1.6) (Problem (1.1)) blow up in a finite time. Indeed, note that

$$
\frac{d}{d t} \int_{0}^{1} u(x, t) d x=u^{q-1}(1, t) \geq\left(\int_{0}^{1} u(x, t) d x\right)^{q-1}
$$

As $q-1>1, u$ can not exists globally, i.e. $T_{\max }<\infty$. To show that $u$ blows up in a finite tine we need to show that $u(1, t) \rightarrow \infty$ if $t \rightarrow T_{\max }$. For smooth solutions of Problem (1.6) satisfying (2.1) it is demonstrated in the proof of Theorem 2 above (for weak solutions of (1.1) see, e.g., [4]).

Remark 3. The regularity of solutions of problems like (1.1) has been intensively studied in [2] (see also the references therein). 


\section{References}

[1] Chipot, M., Fila, M. and P. Quittner: Stationary solutions, blow up and convergence to stationary solutions for semilinear parabolic equations with nonlinear boundary conditions. Acta Math. Univ. Comenianae 40 (1991), 35 - 103.

[2] DiBenedetto, E.: Degenerate Parabolic Equations. New York: Springer-Verlag 1993.

[3] Fila, M. and J. Filo: Blow-up on the boundary: a survey. Singularities and Differential Equations. Banach Center Publications 33 (1996), 67 - 79.

[4] Filo, J.: On solutions of a parturbed fast diffusion equation. Aplikace Maternatiky 32 (1987), $364-380$.

[5] Filo, J.: Diffusivity versus absorption through the boundary. J. Diff. Equ. 99 (1992), 281 $-305$

[6] Filo, J.: Local existence and $L^{\infty}$-estimate of weak solutions to a nonlinear degenerate parabolic equation with nonlinear boundary data. PanAmer. Math. J. 4 (1994)3, $1-31$.

[7] Friedman, A.: Partial Differential Equations of Parabolic Type. Englewood Cliffs: Prentice-Hall 1964.

[8] Friedman, A. and B. McLeod: Blow-up of positive solutions of semilinear heat equations. Indiana Univ. Math. J. 34 (1985), $425-447$.

[9] Levine, H. and L. E. Payne: Nonexistence theorems for the heat equation with nonlinear boundary conditions and for the porous medium equation backward in time. J. Diff. Equ. 16 (1974), $319-334$.

[10] Ladyzenskaja, O. A., Solonnikov, V. A. and N. N. Uralceva: Linear and Quasilinear Equations of Parabolic Type (Translations of Mathematical Monographs: Vol. 23). Providence (Rhode Island): Amer. Math. Soc. 1968.

Received 28.05.1997 